\title{
A Retrospective Study of Malaria Cases Reported in a Decade at Tertiary Level Hospital of Western India
}

\author{
Gaur Kusum $^{1 \S}$, Raj Dilip ${ }^{2}$, Kewalramani Suresh ${ }^{3}$, Sharma Darmesh K. ${ }^{4}$, \\ Sharma Meenakshi ${ }^{5}$ Manohar R. K. ${ }^{6}$ \\ ${ }^{1}$ Professor, Department of Preventive and Social Medicine, SMS Medical College, Jaipur (Rajasthan) India. \\ , 2 Assistant Professor, Department of Preventive and Social Medicine, SMS Medical College, Jaipur \\ ${ }^{3}$ Assistant Professor, Department of Preventive and Social Medicine, SMS Medical College, Jaipur \\ ${ }^{4}$ Assistant Professor, Department of Preventive and Social Medicine, SMS Medical College, Jaipur . \\ ${ }^{5}$ Professor, Department of Physiology, SMS Medical College, Jaipur (Rajasthan) India. \\ ${ }^{6}$ Professor and Head, Department of Preventive and Social Medicine, SMS Medical College, Jaipur \\ ${ }^{\S}$ Corresponding author
}

\begin{abstract}
Background: Malaria, a non-fatal disease if detected promptly and treated properly, still causes many deaths in malaria-endemic countries. The present study is intended to find out changing pattern of malarial morbidity and mortality in western India Methods: A retrospective record base study was conducted on malarial cases reported at medical out-patient door (OPD) of SMS Hospital Jaipur (Rajasthan) during last decade i.e. from $1^{\text {st }}$ Jan 2003 to $31^{\text {st }}$ Dec 2012. Available data regarding socio-demographic and mortality profile was collected and analyzed. Case fatality Rates and Proportional Death rates were found out along with cause of death in malaria cases. Chi-squire test was used to find out the significance of difference between proportions. Results: Out of total 3748 malaria cases, maximum cases were reported in Aug to Oct i.e. 2614 (69.74\%). Mean age of diseases was 37.4 years with 3.2 M:F Ratio. Maximum Case Fatality Rate was reported in 2003 which decreases with time with sum ups and downs and in 2012 it remains only 1.8\%. Most frequent (33\%) cause of death was cerebral malaria. Conclusions: Malaria has seasonal variation with maximum cases in post monsoon season affecting mainly middle aged persons. Although there is no certain trend on malarial morbidity but malarial mortality has significantly declined trend.
\end{abstract}

Keywords: Morbidity, Mortality, Case Fatality Rate, Proportional Death Rate

\section{INTRODUCTION}

Malaria, a non-fatal disease if detected promptly and treated properly, still causes many deaths in malaria-endemic countries. Approximately, $5 \%$ of the world's population is infected, out of total malaria cases mainly reported from African regions (81\%), followed by the South-East Asia (13\%), and the Eastern Mediterranean region (5\%)[1]. In 2010, there were 106 malaria endemic countries with 3.3 billion people at risk with 216 million episodes of malaria cases were reported worldwide[1]. WHO with all associated countries including India plans to eliminate malaria and other diseases by 2015, by implementing millennium development goals [1]. ${ }^{1}$

India contributes about $77 \%$ of total malaria cases in South East Asian Region [2]. Currently $80.5 \%$ of Indian population are living in at risk area for malaria [3]. About $92 \%$ cases and $97 \%$ deaths due to malaria were reported from Orisa, Chhattisgarh, Jharkhand, M. P., A.P., Gujraj and Rajasthan. Urban areas also contribute $15 \%$ of total malaria cases reported in India [4]. Rajasthan reported 50945 malaria cases and 2331 P Falciparum cases with 26 deaths due to malaria during 2011.

Malaria is curable and deaths occur due to complication which can be preventable. Early diagnosis of malaria and treatment can reduce the morbidity and prevent deaths. It also contributes in reducing transmission of the disease.

\section{METHOD}

A retrospective record base study was conducted on malarial cases reported at medical outpatient door (OPD) of SMS Hospital, Jaipur (Rajasthan) during last decade i.e. $1^{\text {st }}$ Jan.2003 to $31^{\text {st }}$ Dec. 2012. Records of malaria patients attended during study period were obtained from the record room of the SMS Hospital. Available information was gathered and complied in the form of master chart in MS excel 2007. Data thus collected were classified and analysed. Chi-square test was used to find out the significance of difference between proportions. For significance 'p' value equals to or less than 0.05 will be considered significant. 


\section{a. Trend of Malarial Morbidity}

\section{RESULTS}

Out of total 3748 malaria cases reported during $1^{\text {st }}$ Jan 2003 to $31^{\text {st }}$ Dec 2012, initialy in 2003, 281 malaria cases were reported to attend at SMS Hospital, Jaipur, which start increasing with some ups and down and become $512(13.66 \%)$ i.e. maximum in year 2008. Suddenly in year 2009 it reduces to 327 cases after that it again start increasing upto 467 in year 2011. (Table No.1) Mean age of cases was 37.4 years with $3.2 \mathrm{M}: \mathrm{F}$ Ratio. Maximum i.e. 1604 (42.8\%) cases were reported in 20-40 years age group.

\section{b. Seasonal Variation Malarial Morbidity}

When seasonal variation was concerned, about two third cases reported from Aug to Oct i.e. 2614 (69.74\%) with maximum cases in the month of September i.e. 1119 (29.8\%). This difference in proportion as per the month was found highly significant $(\mathrm{P}<0.001)$. (Fig 1)

\section{c. Trend of Malarial Mortality}

Among all 216 deaths due to malaria during study period, maximum (about $1 / 3$ i.e. $32.41 \%$ ) were reported in year 2007 and 2008 (35 deaths in each of the month). After that proportion of deaths starts decreasing so and so that in year 2012 there are only 5 deaths due to malaria. This difference in proportion was also found highly significant $(\mathrm{P}<0.001)$.

Although proportional death deaths were maximum in year 2007 and 2008 but Case Fatality Rate was reported maximum (10.32\%) in 2003 which decreases with time with sum ups and downs and in 2012 it remains only $1.8 \%$. This difference in CFRs as per the time was also found highly significant $(\mathrm{P}<0.001)$.

\section{d. Association of Age with Malarial Morbidity and Mortality}

Although maximum proportion of cases and deaths due to malaria was reported in 20-40 years of age group but maximum CFR was observed in cases below 20 years of age and minimum (3.7\%) CFR was observed in 40-60 years of age group. This difference in CFRs as per the age was found significant $(\mathrm{P}=0.002)$.

\section{e. Causes of Malarial Mortality}

Out of 216 total deaths due to malaria during study period, cause of death was unknown in 24 (11.11\%) cases. Cause of death in maximum of cases was cerebral malaria followed by MODS, Liver failure, Cardiopulmonary arrest etc.

\section{Figures: Figure 1 - Line Diagramme - SEASONAl VARIATION OF MAlaRia CASES}

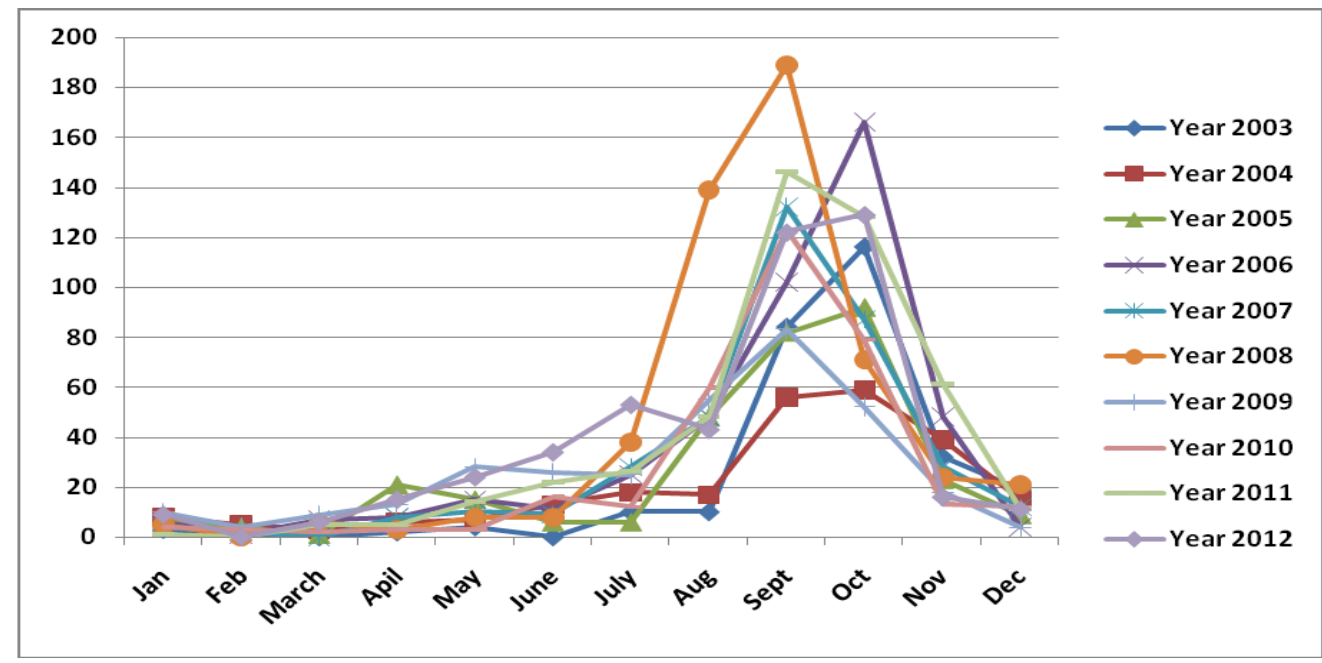


a. Figure 2 - Year-wise malarial morbidity and mortality

\section{Yearwise Component Bar diagram of total malaria cases}

Alive Deaths

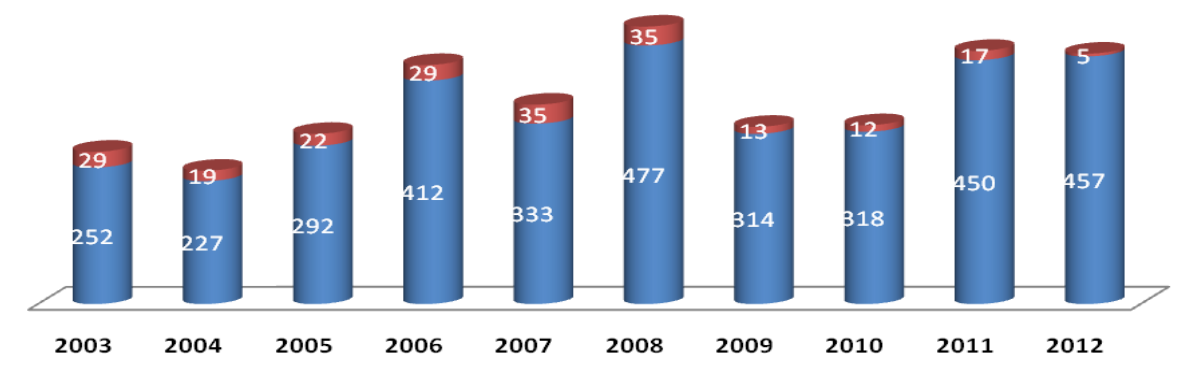

Years

Chi-square $=51.705$ with 9 degrees of freedom; $\quad P<0.001$

$\mathbf{L S}=\mathbf{H S}$

Table No. 1

Year-wise distribution of Malaria Cases and Deaths due to Malaria

\begin{tabular}{|l|l|l|l|l|}
\hline S. No. & Year & Malaria Cases & Malaria Deaths & Case Fatality Rate (CFR in \%) \\
\hline $\mathbf{2}$ & $\mathbf{2 0 0 3}$ & 281 & 29 & $\mathbf{1 0 . 3 2}$ \\
\hline $\mathbf{2}$ & $\mathbf{2 0 0 4}$ & 246 & 19 & 7.72 \\
\hline $\mathbf{4}$ & $\mathbf{2 0 0 5}$ & 314 & 22 & 7.01 \\
\hline $\mathbf{5}$ & $\mathbf{2 0 0 6}$ & 441 & 29 & 6.58 \\
\hline $\mathbf{6}$ & $\mathbf{2 0 0 7}$ & 368 & $\mathbf{3 5}$ & 9.51 \\
\hline $\mathbf{2 0 0 8}$ & $\mathbf{5 1 2}$ & $\mathbf{3 5}$ & 6.84 \\
\hline $\mathbf{8}$ & $\mathbf{2 0 0 9}$ & 327 & 13 & 3.98 \\
\hline $\mathbf{9}$ & $\mathbf{2 0 1 1}$ & 330 & 12 & 3.64 \\
\hline $\mathbf{1 0}$ & $\mathbf{2 0 1 2}$ & 467 & 17 & 3.64 \\
\hline $\mathbf{1 1}$ & Total & 462 & 5 & 1.08 \\
\hline
\end{tabular}

Chi-square test for Malaria Deaths trend from 2003 to $2012=$

Chi-square $=51.705$ with 9 degrees of freedom; $P<0.001$

$\mathbf{L S}=\mathbf{H S}$

Table No. 2

Age-wise distribution of Deaths due to Malaria (total Deaths=216)

\begin{tabular}{|l|l|l|l|l|l|}
\hline S. No. & $\begin{array}{l}\text { Age Group } \\
\text { (Years) }\end{array}$ & Malaria Cases & Malaria Deaths & $\begin{array}{l}\text { Proportional Death } \\
\text { (PDR in \%) }\end{array}$ & $\begin{array}{l}\text { Rate } \\
\text { Case fatality Rate } \\
\text { (PDR in \%) }\end{array}$ \\
\hline $\mathbf{1}$ & $<\mathbf{2 0}$ & 507 & 37 & 17.1 & 7.69 \\
\hline $\mathbf{2}$ & $\mathbf{2 0 - 4 0}$ & $\mathbf{1 6 0 4}$ & $\mathbf{1 0 8}$ & 50 & 6.73 \\
\hline $\mathbf{3}$ & $\mathbf{4 0 - 6 0}$ & 1162 & 41 & 19 & $\mathbf{3 . 7}$ \\
\hline $\mathbf{4}$ & $>\mathbf{6 0}$ & 473 & 30 & 13.9 & 6.55 \\
\hline $\mathbf{5}$ & Total & $\mathbf{3 7 4 8}$ & $\mathbf{2 1 6}$ & $\mathbf{1 0 0}$ & $\mathbf{5 . 7 6}$ \\
\hline
\end{tabular}

Chi-square test for Malaria cases as per age groups $=$

Chi-square $=15.430$ with 3 degrees of freedom; $P=0.002$

$\mathbf{L S}=\mathbf{S}$

Table No. 3

Cause of Death-wise distribution of Deaths due to Malaria (total Deaths=216)

\begin{tabular}{|l|l|l|l|}
\hline S. No. & Cause of Death & Malaria Deaths & $\begin{array}{l}\text { Proportional Cause of Death Rate } \\
\text { (PDR in \%) }\end{array}$ \\
\hline $\mathbf{1}$ & Cerebral Malaria & $\mathbf{4 3}$ & $\mathbf{1 9 . 9 1}$ \\
\hline $\mathbf{2}$ & MODS & 33 & 15.28 \\
\hline $\mathbf{3}$ & Cardio-pulmonary Arrest & 21 & 9.72 \\
\hline $\mathbf{4}$ & Shock & 15 & 6.94 \\
\hline $\mathbf{5}$ & Septicemia/Toxemia & 9 & 4.17 \\
\hline $\mathbf{6}$ & Liver Failure & 25 & 11.57 \\
\hline $\mathbf{7}$ & Renal Failure & 12 & 5.56 \\
\hline $\mathbf{8}$ & Haemorrage & 7 & 3.24 \\
\hline $\mathbf{9}$ & Not Known & 24 & 11.11 \\
\hline $\mathbf{1 0}$ & Total & 216 & \\
\hline
\end{tabular}




\section{DISCUSSION}

In the present study, although maximum cases were reported in year 2008 but difference in proportion of cases with the time was not significant $(\mathrm{p}>0.05)$. Well comparable observations were reported by Yadav R. S. etall, [5], who observed a gradual resurgence of malaria by waves of low and high incidences. Very similar to the present study observations were reported by Mharakuewa S. etall reported malaria cases initially increased from levels in 2003 to a peak in 2008 but then declined from 2008 to 2010 [6]. In contrast to this Anirudh R. etall observed a significant decline in the incidenceby $89.3 \%$ for the last ten years (2001 to 2011) and the percentage of malaria cases shows gradual decrease from the year 2001 to 2011, except in the years 2005 and 2010 [7]. In spite of control measures for malaria since long time there is no significant decrease in cases, this may be because of increase surveillance for malaria and improvement of diagnostic capacity with rapid diagnostic tests (RDTs) [6].

Seasonal variation was also observed in the present study i.e. maximum cases were reported in Agust to Octumber (Post mansoon Season). Seasonal variation was also observed by other authors like Yadav R. S. Etall [5], they also reported seasonal variation, with that of P. vivax increasing from January to September but then declining as the incidence of P. falciparum increased. Well comparable observation were of Gupta etall [8] they also reported that the proportion of $\mathrm{P}$. falciparum malaria cases tends to be greatest during the postmonsoon season while the proportion of P. vivax malaria cases tends to be greatest in the monsoon season [8] In the present study mean age of cases was 37.4 years with $3.2 \mathrm{M}$ :F Ratio and highly significant variation as per age $(p<0.001)$. Almost similar was reported by Anirudh etall [7] that the age group prone to malaria is between 21 to 30 years. In contrast to this Aline Munier etall [9] reported mean age 17.5 years with male/female ratio 0.83. Likewise Yadav R. S. Etall [5] also reported that age-specific differences seen in incidence were not statistically significant $(\mathrm{P}=0.7)$. This difference may be because children upto 15 years reported to J.K..Lone Children hospital attached to this institute and a special adult age group mostly reporting here.

Among all 216 deaths due to malaria during study period, maximum (about $1 / 3$ i.e. $32.41 \%$ ) were reported in year 2007 and 2008 (35 deaths in each of the month). After that proportion of deaths starts decreasing so and so that in year 2012 there are only 5 deaths due to malaria. This difference in proportion was also found significant $(\mathrm{P}=0.011)$. Although proportional death deaths were maximum in year 2007 and 2008 but Case Fatality Rate was reported maximum (10.32\%) in 2003 which decreases with time with sum ups and downs and in 2012 it remains only $1.8 \%$. This difference in CFRs as per the time was also found highly significant $(\mathrm{P}<0.001)$. Almost similar trend of decline in deaths due to malaria was reported by other authors $[5,6,8]$.

Although maximum proportion of cases and deaths due to malaria was reported in 20-40 years of age group but maximum CFR was observed in cases below 20 years of age and minimum (3.7\%) CFR was observed in 40-60 years of age group. This difference in CFRs as per the age was found significant $(\mathrm{P}=0.002)$. Age specific deaths due to malaria were reported maximum after 70 years by many authors $[10,11,12]$.

In the present study cause of death maximum of malaria cases was cerebral malaria followed by MODS, Liver failure, Cardio-pulmonary arrest etc. Other authors also observed change in pattern of clinical representation of sever malaria. More severe complications were observed with the time. Multi-organ failure is more common with Plasmodium falcifarum than Plasmodium vivex malaria [2].

\section{CONCLUSIONS}

Although malaria cases did not have decline trend but deaths due to malaria have certainly significant decline trend. Malaria has seasonal variation with maximum cases in Aug to Oct i.e. post-monsoon season. It affects middle aged population predominately males so may affects economy of the country. Most frequent cause of death was cerebral malaria.

\section{REFERENCES}

[1]. Shuman S: Structure, mechanism, and evolution of the mRNA capping apparatus. Prog Nucleic Acid Res Mol Biol 2000, 66:140

[2]. World Malaria Report 2011,(World Health Organization, Geneva,2012)

[3]. Kumar A, ValechaN, Jain T, Dash AP. Burden of Malaria in India:retrospective and prospective view. Am J Trop Med Hyg 2007, 77 (Suppl 6) 69-78

[4]. Dash A.P., Valecha N, Anvikar A. R. and Kumar A 2008. Malaria in India: Challenges and opportunities; J. Biosci. 33 583-592

[5]. Kumar A, Valecha N, Jain T and Dash A P 2007 Burden of Malaria in India: Retrospective and Prospective View; Am. J. Trop. Med.Hyg. 77 69-78

[6]. Yadav RS, Bhatt RM, Kohli VK, Sharma VP. The burden of malaria in Ahmedabad city, India: a retrospective analysis of reported cases and deaths. Ann Trop Med Parasitol. 2003 Dec; 97(8):793-802

[7]. Mharakurwa S, Mutambu SL, Mberikunashe $J$ etall. Changes in burden of Malaria following scale-up of malaria control interventions in Mutasa district, Zimbabwe. Malar. J. 2013 Jul 1:12:223

[8]. Anirudh R. Acharya et al Trend of malaria incidence in the state of Karnataka, India for 2001 to 2011 Arch. Appl. Sci. Res., 2013, 5 (3):104-111 
[9]. Gupta S, Gunter JT, Novak RJ, Regens JL. Patterns of Plasmodium vivax and Plasmodium falciparum malaria underscore importance of data collection from private health care facilities in India. Malar J. 2009 Oct 12; 8:227

[10]. Aline Munier, Aldiouma Diallo, Adama Marra etall. Evolution of Malaria Mortality and Morbidity after the emergence of chloroquine resistance in Niakhr, Senegal. Malaria Journal 2009, 8:270

[11]. Jha P., Gajlakshmi etall Prospective study of one million deaths in India: Rational, design and validational results. PLoS Med. 2006;3 (Suppl. 2)

[12]. Registrar General of India and Centre for Global Health Review. Cause of deaths in India 2001-2003, SRS, New Delhi: Govt. Of India; 2009

[13]. Gajlakshmi V, etall. Smoking and mortality from tuberculosis and other diseases in India: Retrospective study of 843000 adult male and 35000 controls. Lancet. 2003;362 (Suppl 9383):507-15 